factors, typically to odorous chemicals, but also to non-perceivable factors considered potentially harmful to health, e.g. electrical devices. Reactions due to intolerance range from unpleasant sensations or annoyance to disabling symptoms, which may lead to serious restrictions in daily life. Reactions often initiate exposure assessments in work, home or public environment and may lead to excessive actions to eliminate minor exposures.

In 1996, the WHO classified all medically unexplained conditions attributed to different environmental exposures under the term idiopathic environmental intolerance (IEI), regardless of the factor in question (IPCS 1996). The factors may include e.g chemicals, moulds. The term is most commonly used for multiple chemical sensitivity (MCS), but also nonspecific building-related symptoms (or sick building syndrome), hypersensitivity to electromagnetic fields (EMFs) are regarded as its subtypes. Although the various types of environmental intolerance (EI) share common core characteristics, there is no generally agreed definition of the condition.

The prevalence estimates are based on self-reporting. In adults, the prevalence estimates of EI attributed to chemicals vary from $9 \%$ to $52 \%$, to EMFs from $1.5 \%$ to $21 \%$, and to sounds from $8 \%$ to $39 \%$ (Karvala, et al 2017, submitted).

Environmental intolerance manifests as different degrees of annoyance, which shows a continuum with increasing symptoms, behavioural changes and disability.

IPCS/WHO (1996). Conclusions and recommendations of a workshop on Multiple Chemical Sensitivities (MCS). International Program on Chemical Safety/World Health Organisation. Regul Toxicol Pharmacol 24:188-189.

Keywords: Idiopathic environmental intolerance, reactions to environment, multiple chemical sensitivity, sick building syndrome, sensitivity to electromagnetic fields

\section{6b LOW LEVEL CHEMICAL EXPOSURES - WHY DO SOME INDIVIDUALS DEVELOP HEALTH SYMPTOMS WHEREAS OTHERS DO NOT?}

Anna-Sara Claeson. Department of Psychology, Umeå University, Sweden

10.1136/oemed-2018-ICOHabstracts.813

People are on a daily basis exposed to a variety of odorous and pungent substances. For a person with severe chemical intolerance (CI) or building-related intolerance (BRI) such exposure can result in substantial suffering and reduced quality of life. Symptoms are reported in relation to low-level chemical exposures and there is currently no established doseresponse relationship between exposure to certain compounds and reports of symptoms. Most of the volatile organic compounds (VOCs) identified in indoor air are non-reactive and chemicals that might be more important for symptom reports require specific sampling and analytical methods and are therefore probably not included in the measurements. The overall aim was to investigate the role of individual factors in the development of health symptoms due to exposure to low levels of VOCs.

Data from a cross-sectional field study investigating individuals diagnosed with BRI will be presented as well as data collected from controlled exposures in an exposure chamber. By combining data on individual factors with data from new, more sensitive methods for measurement of organic compounds we are able to study indoor air health problems in a new light. There is a large individual variation in the response to exposures to certain reactive compounds. Exposure-related factors such as type of compound and duration of exposure are of importance. One example is the reactive compound acrolein that induced sensory irritation in a time-dependent manner at a concentration below previously reported detection levels and at half the Swedish occupational threshold limit. Factors related to the individual such as CI, stress or inflammation are also of importance for reports of sensory irritation due to low level chemical exposures. Further, negative affect and information about the exposure also mediate annoyance and symptoms. In order to understand sensory irritation from low-level exposure to VOCs we have to take both individualand environmental factors into account.

\section{$1716 \mathrm{C}$ IS ENVIRONMENTAL INTOLERANCE RELATED TO CHEMICAL OR ODOUR HYPERSENSITIVITY? A SUMMARY OF EPIDEMIOLOGICAL AND EXPERIMENTAL EVIDENCE}

Christoph van Thriel. Leibniz Research Centre for Working Environment and Human Factors, TU Dortmund, Dortmund, Germany

\subsection{6/oemed-2018-ICOHabstracts.814}

Multiple chemical sensitivities (MCS) nowadays more often called idiopathic environmental intolerance (IEI) is characterised by the recurrent occurrences of multiple symptoms involving in multiple organ systems. With respect to the aetiology of IEI toxico-, immune-, and psychogenic theories have been proposed but the exact physiological mechanisms underlying this syndrome are fare from being conclusive. The complex health complaints are triggered by environmental chemicals in very low doses and the upper respiratory tract and the nervous system are two of the most prominent organs of these health complains. Due to the fact that these 'trigger concentrations' are usually tolerated by most persons, genetic (e.g. slower detoxification) and acquired hypersensitivities (odour sensitivity) are thought to be characteristic features of IEI patients. In questionnaires assessing sub-clinical levels of chemical intolerance items addressing odour sensitivity are often used. Our experimental research showed that such scores predict a more unidimensional hedonic evaluation (e.g. malodors) of chemicals. Moreover, differentiation between odours was reduced. In line with other researchers we also showed that such self-descriptions are not associated with better olfactory acuity assessed with standardised psychophysical methods. When combining our own experimental findings with epidemiological studies on IEI we came to the conclusion that chemical or odour hypersensitivity is not an increased sensitivity of chemosensory pathways, but it appears to be a uniform and affective style of responding to low-level exposures to volatile chemicals. Such an undifferentiated response might also explain the fact the chemical triggers of IEI symptoms span from natural fragrances, to perfumes, to industrial chemicals like solvents. 


\section{6d EFFECTS OF OLFACTORY STIMULUS BY ODOUR ON CEREBRAL BLOOD FLOW AND PERIPHERAL BLOOD OXYGEN LEVELS IN MULTIPLE CHEMICAL SENSITIVITY}

${ }^{1} \mathrm{~K}$ Azuma*, ${ }^{2} \mathrm{U}$ Uchiyama, ${ }^{2} \mathrm{M}$ Tanigawa, ${ }^{3} \mathrm{I}$ Bamba, ${ }^{4} \mathrm{M}$ Azuma, ${ }^{5} \mathrm{H}$ Takano, ${ }^{2} \mathrm{~T}$ Yoshikawa, ${ }^{6} \mathrm{~K}$ Sakabe. ${ }^{1}$ Kindai University Faculty of Medicine, Osakasayama, Japan; ${ }^{2}$ Louis Pasteur Centre for Medical Research, Kyoto, Japan; ${ }^{3}$ Tokyo Gakugei University, Koganei, Japan; ${ }^{4}$ Kio University Faculty of Health Sciences, Kitakatsuragi-gun, Japan; ${ }^{5}$ Kyoto University, Kyoto, Japan; ${ }^{6}$ Tokai University School of Medicine, Isehara, Japan

\subsection{6/oemed-2018-ICOHabstracts.815}

Introduction Multiple chemical sensitivity (MCS), often called idiopathic environmental intolerance, is a chronic acquired disorder characterised by nonspecific symptoms attributed to exposure to common odorous chemicals. We previously reported significant activations in the prefrontal cortex (PFC) during olfactory stimulation using several different odorants in patients with MCS compared with controls. Previous clinical observations demonstrated differences in oxygen partial pressure in peripheral venous blood between patients with MCS and controls. Our objective is to investigate peripheral blood oxygen levels during olfactory stimulation to characterise patients with MCS.

Methods We investigated changes in the prefrontal area using near-infrared spectroscopic (NIRS) imaging and those in peripheral arterial blood oxygen saturation $\left(\mathrm{SpO}_{2}\right)$ using a pulse oximeter during olfactory stimulation with odorants $(\gamma$ undecalactone) at three concentrations (zero, odour recognition threshold, and normal perceived odour level) in 11 patients with MCS and 10 controls. We also examined their oxygen partial pressures in peripheral venous blood at normal condition before the test.

Results We observed significant activations in the PFC during olfactory stimulation in patients with MCS. The $\mathrm{SpO}_{2}$ in peripheral arterial blood in patients with MCS remained higher than that in controls during olfactory stimulation tests. The $\mathrm{SpO}_{2}$ remarkably decreased in patients with MCS compared with that in controls during olfactory stimulus at normal perceived odour level and the recovery of the $\mathrm{SpO}_{2}$ after the stimulus was delayed in these patients. No significant differences in oxygen partial pressures of peripheral venous blood were observed.

Conclusion Patients with MCS exhibited stronger brain responses to odours at normally perceived levels. Changes in $\mathrm{SpO}_{2}$ in the peripheral arterial blood suggest that MCS might result in poor oxygen supply to the peripheral tissues or inadequately control the oxygen supply.

\section{6 e MOBILE PHONE USE AND ONSET OF SYMPTOMS. AN UPDATE OF THE EVIDENCE FROM PROSPECTIVE COHORT STUDIES}

L Hillert. Institute of Environmental Medicine, Karolinska Institutet, Stockholm, Sweden

\subsection{6/oemed-2018-ICOHabstracts.816}

Parallel to the rapid increase in mobile phone use worldwide concerns have been raised about various health effects. Acute symptoms have by some individuals been reported to be triggered when holding the phone to the head during calls or being close to a base station for mobile telephony. Symptoms vary, but headache, fatigue and difficulties concentrating are among the most commonly reported. Exposure to radiofrequency fields have been proposed as the cause of the symptoms. Double blind provocation studies have however failed to provide support for such a causal link. In spite of this, some people are still convinced that their ill health is caused by exposure to radiofrequency fields from mobile phones. It has also been suggested that a greater number of people than those who report experiencing these reactions to radiofrequency fields might be affected without being aware of the triggering factor. In theory, long term effects after repeated frequent exposure to radiofrequency fields from mobile phones may exist regardless of whether acute effects exists or not. The first studies on a possible relation between mobile phone use and symptoms in the general population were cross-sectional studies. This study design gives little information on possible causal relationship and suffers from a risk of reverse causality as well as a nocebo effect. Lately however, a number of prospective cohort studies have been published. This presentation will provide an update of the knowledge on mobile phone use and the onset of symptoms from these studies.

\section{$1716 f$ ENVIRONMENTAL INTOLERANCE - A CLINICAL PERSPECTIVE}

${ }^{1,2} \mathrm{M}$ Tondel. 'Occupational and Environmental Medicine, Uppsala University Hospital, Uppsala, Sweden; ${ }^{2}$ Occupational and Environmental Medicine, Department of Medical Sciences, Uppsala University, Uppsala, Sweden

\subsection{6/oemed-2018-ICOHabstracts.817}

Environmental intolerance is relatively prevalent in the general population, recognised by the patients as highly variable symptoms attributed to different perceived harmful exposures such as indoor environment, chemicals, odours, electromagnetic fields and noise. It is not uncommon with progression from a few triggers and symptoms to a multifaceted complex of symptoms triggered by multiple factors. Typically, the symptoms occur at very low exposure levels, below thresholds of acute toxicologic effects and at levels tolerated by a large majority of the population. To understand the background of environmental intolerance, a multi-professional approach is needed with knowledge of allergy, public health, toxicology, physics, chemistry, psychiatry and psychology. In Uppsala we have developed a model where the patients have individual consultations with a medical doctor, an industrial hygienist and a psychologist, respectively. The overall aim is a thorough evaluation of symptoms, exposure and temporal relationship to rule out a medical disease aiming at curative treatment. A well-defined disease is only established in a minority of the cases. Instead, a holistic approach is needed including analysing a range of aspects that may contribute to the condition. The attitude towards the patient, in the professional team, is characterised by active listening, seriousness, confidence building and sufficient time for the patient to describe symptoms and triggers. The final assessment of the patient is made by the physician after discussing the results of the examinations made by other members of the team. By a respectful response aiming for a trustful relationship a discussion can then continue with the patient giving objective information, based on scientific state of the art, related to environmental intolerance. An individual treatment plan includes, if necessary, medical treatment and cognitive behavioural therapy to encourage the 